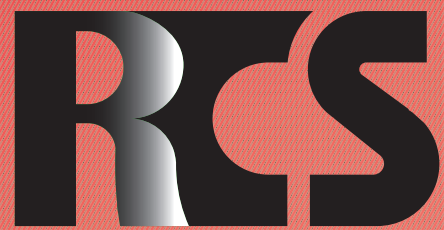

Depósito legal ppi $201502 Z U 4662$

Esta publicación científica en formato digital es continuidad de la revista impresa Depósito Legal: pp $197402 Z$ Z789

- ISSN: 1315-9518 • ISSN-E: 2477-9431

Universidad del Zulia. Revista de la Facultad de Ciencias Económicas y Sociales Vol. XXVI. No.1 


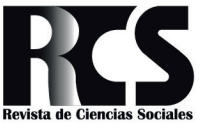

Revista de Ciencias Sociales (RCS). FCES - LUZ

Vol. XXVII, No. 1, 2021, pp. 53-65

- ISSN: 1315-9518 ISSN-E: 2477-9431
Como citar APA: Madariaga, A., Romero, S., Romera, L., y Lazcano, I. (2021). Personas jóvenes con discapacidad y el papel que juega la familia en su ocio. Revista de Ciencias Sociales (Ve), XXVII(1), 53-65.

\title{
Personas jóvenes con discapacidad y el papel que juega la familia en su ocio*
}

\author{
Madariaga, Aurora** \\ Romero, Sheila*** \\ Romera, Liana**** \\ Lazcano, Idurre ${ }^{* * * * * *}$
}

\section{Resumen}

Existen multitud de investigaciones que analizan la realidad del ocio de las personas jóvenes, sin embargo, no son tan abundantes si el foco de atención se centra en el colectivo de jóvenes con discapacidad. El objetivo del presente artículo radica en conocer el ocio de las personas jóvenes con discapacidad y analizar el papel que juega la familia en el mismo. El tipo de investigación es descriptivo, aplicando una encuesta a 400 jóvenes de 15 a 29 años con discapacidad. Los resultados muestran que la juventud con discapacidad, al igual que el resto de la población joven, valora el ocio como elemento importante en sus vidas y que ellos mismos lo organizan, jugando la familia un papel secundario. La presencia explícita de la familia es menor que en otros periodos vitales, y lejos de ser promotora en la organización del ocio del colectivo joven, la familia va disminuyendo su presencia a medida que la persona con discapacidad crece y tiene más autonomía, y por tanto muestra mayor protagonismo en la toma de decisiones. Se concluye, que en las personas jóvenes el ocio es considerado como un espacio vital adecuado para forjar nuevas amistades, y como ámbito de desarrollo personal.

Palabras clave: Ocio; familia; discapacidad; asociaciones; participación.

\footnotetext{
Este texto presenta los resultados relacionados con el papel de la familia en el ocio, que forman parte de una investigación de carácter integral "El ocio de la juventud con discapacidad", financiado por el Gobierno Vasco (España).

** Doctora en Ocio y Desarrollo Humano. Máster en Educación Especial. Experta en Pedagogía Terapéutica. Psicóloga. Docente Investigadora de la Universidad de Deusto, España. E-mail: aurora. madariaga@deusto.es (iD) ORCID: https://orcid.org/0000-0002-8369-2780

**** Doctora en Ocio y Desarrollo Humano. Máster en Dirección de Proyectos de Ocio. Experta en Estadística Aplicada a la Salud. Socióloga. Investigadora en la Fundación GaituzSport Fundazioa, España y colaboradora con el Instituto de Estudios de Ocio de la Universidad de Deusto, España. E-mail: romero.sheila@gaituzsport.eus ORCID: https://orcid.org/0000-0002-8086-4778

***** Profesora Titular da Universidade Federal do Espirito Santo, Brasil. E-mail: liromera@uol.com.br (iD ORCID: https://orcid.org/0000-0003-4809-2744

Doctora en Ocio y Desarrollo Humano. Docente investigadora en el Instituto de Estudios de Ocio de la Universidad de Deusto. España. E-mail: ilazkano@deusto.es (iD ORCID: https://orcid.org/0000-0002$\underline{6746-0357}$
}

Recibido: 2020-09-15 


\title{
Young people with disabilities and the role that the family plays in their leisure
}

\begin{abstract}
There are many studies that analyze the reality of leisure for young people, however, they are not so abundant if the focus of attention is focused on the group of young people with disabilities. The objective of this article is to understand the leisure activities of young people with disabilities and analyze the role that the family plays in it. The type of research is descriptive, applying a survey to 400 young people aged 15 to 29 with disabilities. The results show that young people with disabilities, like the rest of the young population, value leisure as an important element in their lives and that they organize it themselves, with the family playing a secondary role. The explicit presence of the family is less than in other vital periods, and far from being a promoter in the organization of leisure for the young group, the family decreases its presence as the person with a disability grows and has more autonomy, and therefore shows greater leadership in decisionmaking. It is concluded that in young people, leisure is considered as an adequate vital space to forge new friendships, and as an area of personal development.
\end{abstract}

Keywords: Leisure; family; disability; associations; participation.

\section{Introducción}

La investigación sobre la experiencia de ocio ha sido estudiada por diferentes teóricos, como Neulinger (1980), que aborda variables tanto sociales como culturales, o Iso-Ahola (1980), que entiende la experiencia de ocio como un estado mental subjetivo, resultado de la libre elección y llevada a cabo por razones intrínsecas. Sin embargo, también se hace necesario considerar que el ser humano recibe la influencia de la sociedad y del medio en el que vive a la hora de experimentar cualquier vivencia, incluida la de ocio. En este caso, se entiende que ésta se refiere a un estado mental que se deriva de la interrelación entre el participante y su entorno; un ambiente en el que inciden las variables sociales y/o situacionales. Lograr que la vivencia de ocio sea una experiencia valiosa implica cambios conceptuales, actitudinales y estructurales, así como supone asumir, de acuerdo con Cuenca (2003), que frente a un ocio consumo, hay que trabajar un ocio experiencial.

Tomando como referencia la estructura de la oferta de ocio en la realidad actual (Lazcano, Madariaga y Lázaro, 2014; Madariaga, Lazcano y Doistua, 2014), se entiende la experiencia de ocio como una realidad que se alimenta de la vertiente objetiva (sujeto, grupo, actividad, tiempo, espacio y recursos) y de la subjetiva (beneficios, intereses y opiniones, motivaciones y valores).

Para analizar la experiencia de ocio se parte del análisis de la práctica, entendiendo que el individuo otorga definiciones subjetivas a las actividades que practica. De ahí que resulte indispensable añadir otras dimensiones que completen la aproximación conductual. Así, la investigación se complementa con una aproximación a la dimensión cognitiva, centrada en el análisis de las opiniones y preferencias que una persona tiene con respecto a una práctica con la finalidad que ésta se convierta en una experiencia de ocio (Madariaga y Lazcano, 2014).

Asimismo, se analiza la dimensión motivacional, entendiendo que las emociones, además de organizar la cognición y la acción, poseen un efecto motivacional. Se incluye así el elemento con mayor componente intrapsíquico del concepto de estilo de vida: Las motivaciones. Al respecto, sostienen Camargo, Castañeda y Segura (2020), que: "El interés por estudiar variables psicológicas positivas relacionadas con la salud mental ha venido 
en aumento en las últimas décadas, es así como ha destacado entre estas el sentido de la vida" (p.208), relacionada positivamente con la calidad de vida; con lo cual manifiestan Berrios-Riquelme, et al. (2019) que en la cual "convergen los componentes afectivos, emocionales y cognitivos del bienestar subjetivo" (p.132). Por último, se aborda la dimensión cultural; esta aproximación incluye un análisis sociológico o sociocultural, que analiza el consumo de ocio como un acto social y un evento cultural.

A continuación, se describirá el papel de la familia en el desarrollo de toda persona y que se extiende al ámbito del ocio, siendo este la finalidad última del trabajo presentado. La familia es el grupo básico más importante, el primer contexto socializador y el entorno natural en donde los miembros que la forman evolucionan y crecen a nivel afectivo, físico, intelectual y social. Cada familia, es un sistema abierto (Ajuriaguerra, 1980; Lila, Musitu y Buelga, 2000), en continuo movimiento, cambio y reestructuración. Es un entorno interactivo y cualquier suceso que ocurra a alguno de los miembros repercute de una $u$ otra manera sobre los demás; produciendo cambios determinantes en la unidad familiar.

Las experiencias que se adquieren en la primera infancia, de cualquier tipo, y los vínculos de apego que se dan en ella determinan la vida de cada persona. La familia debe ofrecer oportunidades para desarrollar aquellas habilidades y competencias personales y sociales que permitan a sus miembros crecer con seguridad y autonomía en todos los ámbitos de la vida, también en el ocio (Peralta y Arellano, 2010), siendo capaces de relacionarse y de actuar adecuadamente en la sociedad (Ajuriaguerra, 1980).

En la mayoría de las ocasiones, ante determinados hechos que desestabilizan el sistema familiar, se producen desadaptaciones y desajustes en su seno. Una de ellas surge cuando hay un hijo con necesidades especiales derivadas de una discapacidad adquirida antes de nacer, durante el nacimiento o a lo largo de la infancia (Ortega, et al., 2010). El acontecimiento suele ser impactante y repercute, probablemente, a lo largo de toda la vida en la unidad familiar, e incide en el ocio también. Al respecto, Molina, Mateos y Violant (2015), sostienen que en estos casos un escenario de desprotección por parte de la familia, puede conllevar a desarrollar actitudes y comportamientos poco saludables que pudieran reducirse mediante el apoyo de esta.

El afrontar el diagnóstico de discapacidad en un hijo implica de por vida un proceso abierto, por ello los padres experimentan diferentes emociones y etapas. Ligado a cada ciclo vital, la familia vive crisis donde se experimentan de nuevo emociones y se reviven las fases de ajuste (shock, reacción y adaptación), todas las familias pasan por las tres fases en diferentes ocasiones y sobre todo en los tránsitos vitales de una etapa a otra, independientemente de las características o factores determinantes en cada familia (Soto, et al., 2015). Cada familia tiene sus propias características y sus formas de participación en la sociedad y eso hay que respetarlo (Sánchez, 2006).

La familia con un hijo con discapacidad desempeña las mismas funciones que las demás: Tareas encaminadas a satisfacer las necesidades colectivas y personales de los miembros en todos los ámbitos de la vida (salud, educación, vivienda, ocio, entre otros). Según Arnaiz (2004), éstas se pueden desglosar en nueve funciones: Económica; de cuidado físico; de descanso y recuperación; socialización; autodefinición; afectividad; orientación; de educación y vocacional. La diferencia está en que cada una de ellas es más difícil de cumplir cuando se trata de atender a la persona con discapacidad, pues los recursos y apoyos de todo tipo se hacen más necesarios $\mathrm{y}$, en ocasiones, deben ser permanentes.

La articulación de los conceptos familia y discapacidad en la actualidad, está mediada por el contexto social en que los miembros de dichas familias se encuentran inmersos, de tal forma que la representación que cada familia tiene de la discapacidad, si bien, depende en buena medida de las características propias del grupo familiar (Córdoba y Soto, 2007), también refleja de alguna manera el contexto 
de las actitudes sociales y la realidad dentro de la cual dicha representación tiene lugar.

La investigación en el tema de familia y discapacidad se ha reorientado en función del nuevo papel que cumple el sistema familiar. Desde este contexto, Ferguson (2002) realizó una síntesis de los principales hallazgos presentados por los investigadores en el área durante los últimos años:

a. Han incrementado los estudios en torno a que los patrones de ajuste general y bienestar familiar son similares entre grupos de familias de niños con y sin discapacidad. Sin embargo, hay algunas diferencias en el ajuste de la familia de las personas con discapacidad a lo largo de su ciclo vital.

b. Se reconocen en mayor medida los aspectos positivos derivados de la crianza de una persona con discapacidad en el seno familiar. Se han identificado un mayor desarrollo en habilidades de afrontamiento (adaptabilidad), armonía familiar (cohesión), crecimiento espiritual, compartir roles parentales y comunicación.

c. Hay actitudes más presentes en familias en las que existe un miembro con discapacidad, según Zulueta y Peralta (2008) tales como: Saber priorizar, tolerancia, amplitud de miras, el valor de lo cotidiano, empatía, paciencia, solidaridad y motivación.

d. Se ha avanzado en la comprensión de por qué algunas familias son más resistentes que otras en la adaptación al estrés que conlleva el que uno de los miembros de la familia tenga una discapacidad.

e. Algunos estudios sugieren que factores como niveles de discapacidad (discapacidad intelectual con apoyo generalizado) o estructura familiar (padres solos, tamaño de la familia), pueden no ser tan críticos como otros factores (presencia o ausencia de auto-agresión o comportamientos desafiantes, ingresos familiares), en el momento de determinar los niveles de ajuste de la familia a la discapacidad (Córdoba y Soto, 2007).

En el contexto actual se avala la importancia de que la persona con discapacidad ejerza sus derechos, y se desarrolle lo más plenamente posible experimentando distintas posibilidades, siempre con el apoyo y ayuda que cada persona requiera. Por ello, es fundamental que la familia reciba ayuda en los siguientes aspectos o niveles de intervención: Comunicación-información, formación, acompañamiento y asesoramiento a la familia (Blanco, 2008). Actualmente la intervención en familias con un miembro con discapacidad, se transforma de un enfoque patológico de tipo médico a una visión contextual y social del sistema familiar, en la que emergen nuevas y diferentes aproximaciones a la familia; según Córdoba y Soto (2007), destacan estudios de aspectos tales como: Capacitación funcional (empowerment), bienestar familiar (family wellness), ajuste dinámico (resilience) y calidad de vida familiar (family quality of life).

Así pues, un entorno familiar que propicie la autodeterminación debería caracterizarse por el establecimiento de normas y límites claros y, a la vez, por la existencia de cierta flexibilidad para adaptarse a los cambios (Martínez, et al., 2010). Para ello, es necesario:

a. Identificar las necesidades de la familia: En un clima de confianza que permita a las familias expresar sus preferencias y decir todo lo que les preocupa. Así, podrán traducir las preocupaciones de las mismas en necesidades. Papel de las familias soporte, necesidad de apoyo y cuidado; el contexto, requiere el desarrollo de la persona en el ámbito de expresar afectividad así como control conductual y disciplina (Peñafiel, 2012).

b. Identificar los recursos y las fortalezas de la familia: Se parte de la identificación de los aspectos positivos del funcionamiento familiar para poder comprender la realidad y comprobar cómo gestionan y controlan sus propias necesidades. Positividad, cercanía, naturalidad, generar recuerdos, cubrir necesidades, y construir un futuro seguro y cubierto (Fresnillo, 2014).

c. Identificar fuentes de ayuda: Se trata de descubrir con qué apoyos formales o informales (servicios o redes sociales) cuenta la familia, y de qué modo cubren sus necesidades (Álvarez y García, 2007). La familia participa en la red de servicios, en cursos de formación 
$\mathrm{y}$ en generar espacios educativos, todo ello ayuda a afrontar la situación y aplicar la matriz de demandas recursos y percepción de estos (Barrientos, 2013). En este escenario de intervención es fundamental capacitar a las familias. La clave está en crear oportunidades para que todos y cada uno de los miembros de la familia sean más competentes, independientes y autosuficientes (Wehmeyer y Field, 2007). Las familias pueden aprender lo que no saben y desarrollar estrategias así como destrezas que les ayuden en sus tareas, en sus futuros problemas o en sus necesidades.

Por último, Cezar, et al. (2016) estudiaron la importancia de los roles ocupacionales de las personas con discapacidad, entendiendo que el desempeño puede favorecer la mejora de su condición de salud, la construcción de ciudadanía y el aumento de la participación en la vida social. Desempeñar roles también significa para estas personas ampliar sus relaciones sociales y sentirse más independientes en las actividades que desean y les gustaría realizar.

Dada la importancia que ejerce la familia en el colectivo de la persona joven con discapacidad y por tanto en su ocio, y dada la escasez de estudios en los que se aborde este aspecto, el objetivo principal de este estudio consiste en realizar una aproximación al papel que ejerce la familia en el ámbito del ocio del colectivo joven con discapacidad.

\section{Metodología}

La investigación desarrollada es descriptiva de campo. Para recoger los datos de los participantes se realizó una encuesta sobre prácticas y estilos de vida en ocio de la juventud con discapacidad, administrada a jóvenes de 15 a 29 años, lo que proporciona la información que permite conocer la realidad del ocio del colectivo, en una aproximación tanto objetiva: Actividades, prácticas y consumos, tiempos, espacios y recursos; como subjetiva: Motivaciones, intereses, opiniones, valores, preferencias, beneficios y barreras (Madariaga y Romero, 2016).
Para un nivel de confianza del 95\% y un $5 \%$ de error, el tamaño muestral debía ascender a 400 individuos, teniendo en cuenta que la población total era infinita. De cara a distribuir la muestra de un modo equitativo, se procedió a la estratificación de la misma en función de: Tipo de discapacidad, sexo y edad. Quedando distribuida, proporcionalmente, de la siguiente manera: Discapacidad física $(25,3 \%)$, visual $(25 \%)$, auditiva $(25 \%)$ e intelectual $(24,8 \%)$. El $45 \%$ de los jóvenes se sitúan en el intervalo de edad de los 25 a los 29 años de los cuales el $48,8 \%(n=195)$ son mujeres.

Para la recolección de los datos, se acudió a asociaciones de discapacidad auditiva, física, intelectual y visual para cumplimentar los cuestionarios; el acceso a la muestra de jóvenes con discapacidad se completó visitando centros ocupacionales y especiales de empleo ligados a los diferentes tipos de discapacidad, así como institutos, para acceder a la muestra de jóvenes.

Una vez establecidos los contactos con los responsables de las asociaciones y los centros de empleo, se les explicó la finalidad del estudio, la confidencialidad de los datos y se les pidió la participación voluntaria de los jóvenes con discapacidad para responder a un cuestionario en formato papel, solicitando las pertinentes autorizaciones paternas en todos aquellos casos que, a pesar de ser mayores de edad, la tutela recae en los padres o adultos del entorno familiar.

\section{Resultados y discusión}

El análisis de los resultados será descriptivo y éstos se mostrarán a partir de las vertientes objetiva y subjetiva de la experiencia de ocio. Previamente, y para conocer el ocio de los jóvenes, respecto a la actividad principal del mismo, la mayoría realizan alguna actividad deportiva $(\mathrm{n}=287)$, efectuando solamente el $3,5 \%(n=14)$ alguna labor turística durante su tiempo libre, tal y como se puede observar en la Figura I. El deporte es una de las actividades más practicadas y más demandadas por los jóvenes con discapacidad, tanto en su 
Madariaga, Aurora; Romero, Sheila; Romera, Liana y Lazcano, Idurre

Personas jóvenes con discapacidad y el papel que juega la familia en su ocio

dimensión individual como en la opción de papel de la familia es residual. practicarlo en equipo, en ambas prácticas el

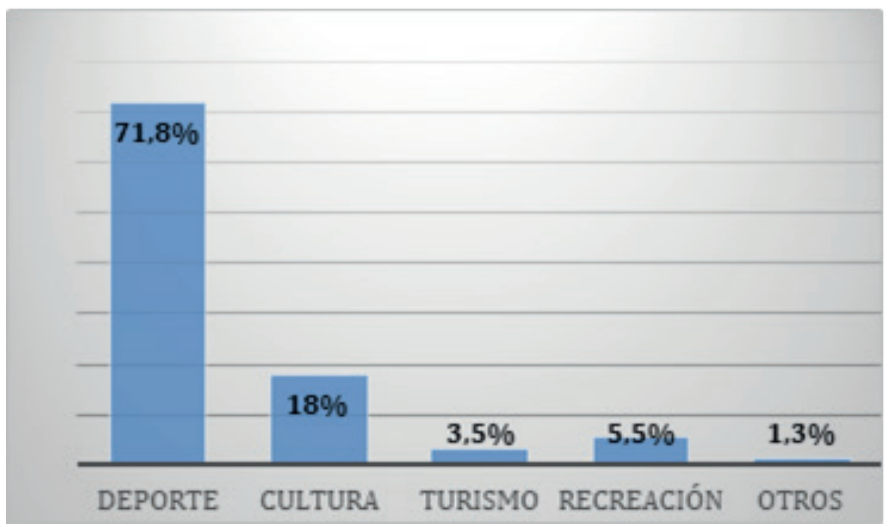

Fuente: Elaboración propia, 2020.

Figura I: Actividad principal de ocio

\subsection{Aproximación objetiva}

Respecto a la aproximación objetiva y el papel que juega la familia en el ocio de los jóvenes se han tenido en cuenta a los organizadores, es decir, qué agentes organizan el ocio de los jóvenes, la compañía, la frecuencia de las prácticas, el lugar de las mismas, los tiempos y los consumos. Tal y como se muestra en la Figura II, prácticamente el $46 \%$ de los jóvenes afirma que su práctica principal de ocio es organizada por sí mismos. La familia, es el agente que juega un papel menor en este caso puesto que solamente el $12,3 \%$ de los jóvenes afirma que es ésta la que desempeña el rol principal en la organización del ocio de sus hijos.

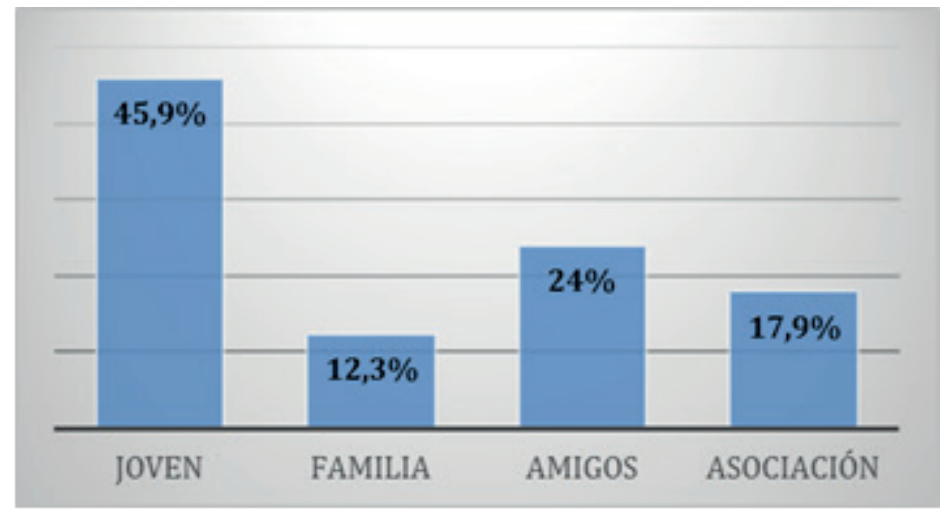

Fuente: Elaboración propia, 2020.

Figura II: Organización de actividades 
Si se desglosa este dato por ámbito de ocio (ver Figura III), se observa que la familia no desempeña un papel primordial en ninguno de los ámbitos analizados. Respecto a otras actividades de ocio (categoría otros), tales como colaborar con tu asociación o hobbies específicos (trenes, cocina, plantas, entre otros), son organizados exclusivamente por las asociaciones a las que pertenecen los jóvenes y que cada una de ellas está vinculada a un tipo de discapacidad.

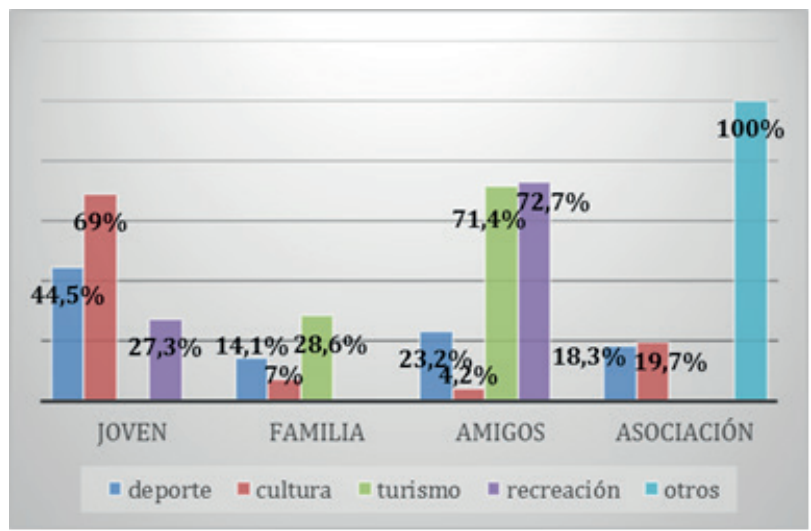

Fuente: Elaboración propia, 2020.

Figura III: Organización de actividades por ámbito de ocio

Además, tal y como se observa en la Figura IV, cabe destacar que son las mujeres jóvenes con discapacidad las que comparten en mayor medida sus actividades de ocio con la familia $(59,5 \%)$, mientras que los hombres las comparten con otros agentes $(52,6 \%)$ como son los amigos (con y sin discapacidad, asociaciones, organizaciones, entre otros).

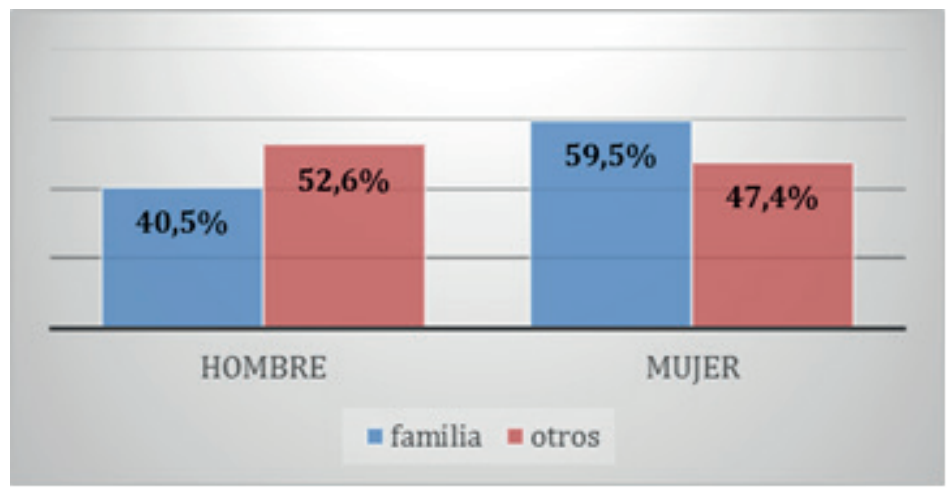

Fuente: Elaboración propia, 2020.

Figura IV: Género y compañía en las prácticas de ocio 
Madariaga, Aurora; Romero, Sheila; Romera, Liana y Lazcano, Idurre

Personas jóvenes con discapacidad y el papel que juega la familia en su ocio

En cuanto a la frecuencia de las prácticas de ocio realizadas (ver Figura V), la familia tiene un papel principal cuando las actividades se realizan de manera más esporádica como una o dos veces al mes $(2,5 \%)$ o en periodos vacacionales $(7,7 \%)$. Cuándo la frecuencia de la práctica aumenta el papel de la familia pierde protagonismo. Destaca el hecho de que cuando la práctica es una vez a la semana juega el mismo papel la familia y los otros agentes analizados (amigos con discapacidad, sin discapacidad, el propio joven y las asociaciones).

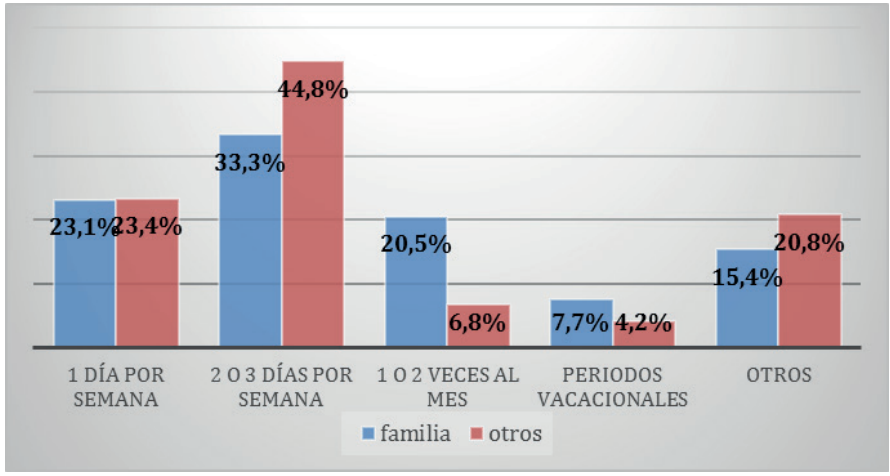

Fuente: Elaboración propia, 2020.

Figura V: Frecuencia en función de la compañía

Respecto al lugar de ejecución de la actividad, tanto la familia como otros agentes realizan las prácticas de ocio con los jóvenes en la comunidad. Como cabía esperar, en casa, la familia también desempeña un rol protagonista (ver Figura VI).

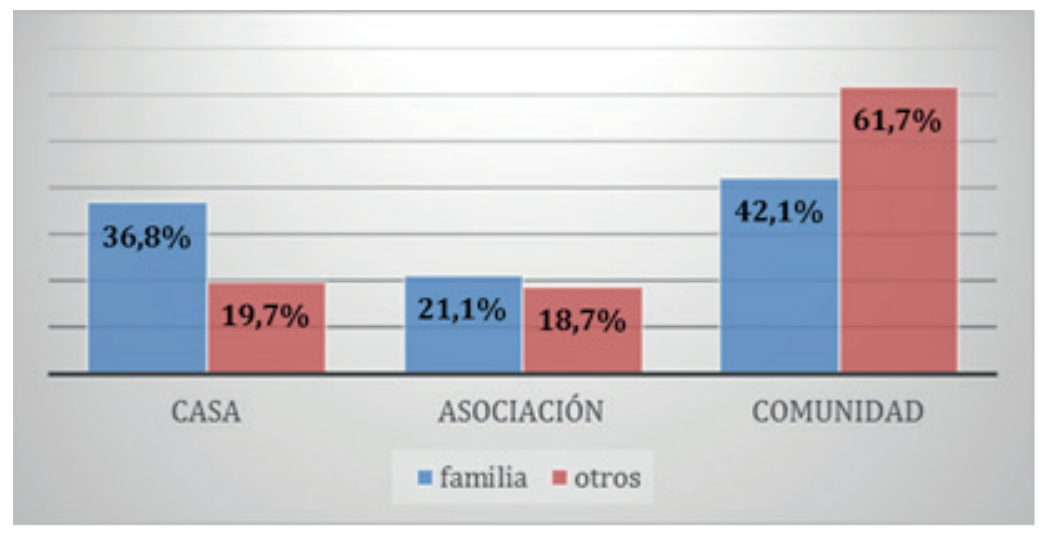

Fuente: Elaboración propia, 2020.

Figura VI: Lugar de realización y compañía de práctica 
En la vertiente objetiva, se ha descrito qué actividad de ocio realizan en mayor medida, destacando la práctica deportiva y además se ha conocido el agente gestor o promotor de la actividad de ocio, variable en la que destaca su autogestión y en la que la familia tiene un papel residual, excepto en algunas actividades de carácter turístico en las que se entiende que se desarrolla, por ejemplo, un viaje familiar. Aunque los amigos también organizan con un papel destacado actividades turísticas y recreativas, mientras tanto la asociación se centra más en actividades culturales y/o deportivas.

Con relación a la compañía con la que realizan la práctica, ellos solos, con amigos con o sin discapacidad y con asociaciones, la familia tiene presencia pero en cuarto lugar y aparece más implicada cuando el joven con discapacidad es mujer que cuando es varón. Si se detiene en la frecuencia, la familia actúa de forma más esporádica y amplía su presencia en periodos vacacionales en los que hay menor oferta de ocio comunitaria y asociativa. El espacio comunitario de acuerdo con Cuenca (2003), es el esencial pero actividades de ocio que se realizan dentro del hogar, lógicamente, están más vinculadas a la presencia de la familia.

\subsection{Aproximación subjetiva}

Si se atiende a la vertiente subjetiva del joven, es decir, a la percepción que este tiene respecto a sus actividades de ocio y el papel que juega la familia en ellas, se puede observar como existe una mayor satisfacción con las actividades de ocio si éstas se realizan con otros agentes sociales (las opciones de respuesta en este caso han ido de 1 a 5). Sin embargo, resulta necesario matizar que la desviación típica de las familias es muy elevada, lo que indica que en este caso también ha habido respuestas muy satisfactorias (ver Tabla 1).

\section{Tabla 1}

Satisfacción con la actividad

\begin{tabular}{lllll}
\hline & Familia & \multicolumn{2}{c}{ Otros } \\
& M & D.E & M & D.E \\
\hline Satisfacción con la actividad & 3.96 & 1.50 & 4.19 & .94 \\
\hline
\end{tabular}

Fuente: Elaboración propia, 2020.

En cuanto a la percepción subjetiva de los jóvenes con discapacidad (Madariaga y Romero, 2016; Lazcano, et al., 2014; Madariaga y Lazcano, 2014; Romero, Madariaga y Lazcano, 2014), éstos están satisfechos con sus prácticas de ocio, y aunque valoran positivamente la presencia de otros agentes (ellos mismos, amigos con y sin discapacidad, asociaciones), también valoran la presencia de la familia en el patrón de conducta de ocio con satisfacción.

En este sentido, dado los hallazgos encontrados se evidencia que a lo largo de los años ha ido evolucionando el modo en el que se concibe a las personas con discapacidad (Portacio-Díaz, Sahagún-Navarro y ValesHidalgo, 2020), por lo que cada vez son más las investigaciones centradas en este colectivo. La novedad de esta investigación radica en la realización de una aproximación a las experiencias de ocio de los jóvenes con discapacidad desde una vertiente objetiva y subjetiva (Madariaga y Romero, 2016; Lazcano, et al., 2014; Madariaga y Lazcano, 2014; Romero, et al., 2014), teniendo en cuenta el papel que juega la familia en ellas.

Tal y como se ha presentado en la introducción, la familia forma parte de la vida 
de toda persona y eso lleva implícito que esté presente en el ámbito del ocio, aunque su papel es muy intenso y permanente en la infancia y va desdibujándose en posteriores etapas vitales, en las que la persona va configurando su identidad como joven y posteriormente como adulto (Peralta, 2008; Peralta y Arellano, 2010; Martínez, et al., 2010). En esas edades la necesidad de formar parte del grupo y de practicar el ocio con amigos es esencial, por ello la familia tiene menor presencia.

SI bien es cierto que en el caso de los jóvenes con discapacidad las familias permanecen de forma más explícita en todos los ciclos vitales, suele ser por su propia necesidad de cuidar o vigilar directamente al joven (Ortega, et al., 2010; Peñafiel, 2012); hay estudios que hablan de conducta de sobre protección, o también se debe a las características propias de la persona con discapacidad (Sánchez, 2006; Álvarez y García, 2007; Soto, et al., 2015), que necesita más ayuda y apoyo al presentar conductas menos autónomas e independientes.

Este hecho se acrecienta en el caso de las mujeres jóvenes con discapacidad. En la actualidad, se incide especialmente en la importancia de dotar de habilidades y herramientas la joven con discapacidad, con la finalidad de que su conducta sea lo más auto determinada posible (Hodapp y Fidler, 1999; Grigal, et al., 2003; González, 2006; Wehmeyer y Field, 2007; Zulueta y Peralta, 2008), aplicable a todos los ámbitos de su vida, incluido el del ocio.

El papel de la familia para que el joven con discapacidad participe en una actividad de ocio que se oferta en la comunidad, es sólo de acompañarle y muy raramente de participar de forma conjunta (Ferguson, 2002; Córdoba y Soto, 2007; Fresnillo, 2014). Lo mismo ocurre en el caso de las asociaciones, con la peculiaridad de que en estas últimas son las juntas directivas formadas en muchos casos por familiares (padres, madres y/o hermanos $\mathrm{y}$ hermanas) las que marcan directrices sobre la oferta de ocio de las asociaciones, el papel del joven con discapacidad e incluso el papel de la familia. En este contexto, la familia mayoritariamente acompaña al joven al lugar donde se celebra la actividad de ocio y ocasionalmente la asociación organiza actividades abiertas para que participen junto a los jóvenes con discapacidad sus familias.

\section{Conclusiones}

Los resultados muestran que la juventud con discapacidad, al igual que el resto de la población joven, valora el ocio como elemento importante en sus vidas y que ellos mismos organizan su ocio, jugando la familia un papel secundario. Asimismo, en las personas jóvenes el ocio es considerado como un espacio vital adecuado para forjar nuevas amistades, y como ámbito de desarrollo personal. La presencia explícita de la familia es menor que en otros periodos vitales, y aunque pueda considerarse que ésta está más presente que en el ocio de las personas jóvenes sin discapacidad, lejos de ser promotora en la organización del ocio del colectivo joven, la familia va disminuyendo su presencia a medida que la persona joven con discapacidad crece y tiene más autonomía, y por tanto, se convierte en protagonista de su toma de decisiones.

A lo largo de los años ha ido evolucionando la manera en la que se concibe a las personas con discapacidad, por lo cual cada vez se desarrollan investigaciones relacionadas en este colectivo. Lo novedoso de esta investigación reside en la realización de una aproximación a las experiencias de ocio de los jóvenes con discapacidad desde una vertiente objetiva y subjetiva; la primera, abarca las actividades, prácticas y consumos, tiempos, espacios y recursos; en tanto que la subjetiva, comprende las motivaciones, intereses, opiniones, valores, preferencias, beneficios y barreras, de este colectivo.

En cuanto a la indagación de la vertiente objetiva, destacaron las prácticas deportivas y además se ha conocido el agente gestor o promotor de la actividad de ocio, variable en la que enfatiza su autogestión y en la cual la familia tiene un papel residual, a excepción de algunas actividades de carácter turístico, 
como la realización de un viaje familiar. En ese sentido, los amigos también organizan con un papel destacado actividades turísticas y recreativas, en tanto que, la asociación se centra más en actividades culturales y/o deportivas.

En cuanto al abordaje subjetivo del joven, es decir, a su percepción con respecto a las actividades de ocio que realiza y el papel que juega la familia en ellas, se pudo apreciar que existe una mayor satisfacción con las actividades de ocio si éstas se llevan a cabo con otros agentes sociales.

Finalmente, se puede concluir que, si en posteriores estudios se comparase el papel de la familia en el ocio de los jóvenes, esta vez, con y sin discapacidad y por tanto se evidenciara diferencias inapreciables, lo mismo supondría un gran avance en materia de inclusión en el ocio de toda la ciudadanía.

\section{Referencias bibliográficas}

\section{Ajuriaguerra, J. (1980). Manual de psiquiatria} infantil. Masson, S.A.

Álvarez, L., y García, J. N. (2007). Las redes de apoyo social en las dificultades del desarrollo. En J. N. García (Coord.), Dificultades del desarrollo. Evaluación e intervención (pp. 317325). Pirámide.

Arnaiz, P. (2004). La educación inclusiva: Dilemas y desafíos. Educación, desarrollo y diversidad, 7(2), 25-40.

Barrientos, P. (2013). La participación familia-escuela en México desde un enfoque inclusivo: Reflexiones sobre familia y discapacidad. Revista Latinoamericana de Educación Inclusiva, 7(2), 175-193.

Berrios-Riquelme, J., Frias-Navarro, D., Pascual-Soler, M., y Muzatto-Negron, P. (2019). Escala de satisfacción con la vida: Propiedades psicométricas de inmigrantes en España. Revista de
Ciencias Sociales (Ve), XXV(1), 130142

Blanco, M. R. (2008). Construyendo las bases de la inclusión y la calidad de la educación en la primera infancia. Revista de Educación, (347), 33-54.

Camargo, J. A., Castañeda, J. G., y Segura, D. P. (2020). Sentido de vida, depresión y ansiedad en población rural de Cundinamarca, Colombia. Revista de Ciencias Sociales (Ve), XXVI(4), 206216. https://dx.doi.org/10.31876/rcs. v26i4.34658

Cezar, D. M., Figueiredo, V., Ferigato, S., y Agostini, R. (2016). Personas con discapacidad y sus roles ocupacionales: trabajo, familia, independencia y participación social. Revista Chilena de Terapia Ocupacional, 16(2), 107117. https://doi.org/10.5354/0719$\underline{5346.2016 .44755}$

Córdoba, L., y Soto, G. (2007). Familia y discapacidad: Intervención en crisis desde el modelo ecológico. Psicología Conductual, 15(3), 525-542.

Cuenca, M. (2003). El ocio como experiencia de desarrollo humano, un reto para el siglo XXI. En C. De la Cruz (Ed.). Los retos del ocio y la discapacidad en el siglo XXI (pp. 33-66). Universidad de Deusto.

Ferguson, P. (2002). A place in the family: An historical interpretation of research on parental reactions to having a child with a disability. The Journal of Special Education, 36, 124-130. https://doi.org/10.1177/002246690203 $\underline{60030201}$

Fresnillo, M. (2014). Desde la familia, la discapacidad se mira con otros ojos. Revista Educación y Futuro Digital, (9), 60-73.

González, M. C. (2006). La motivación y el autoconocimiento como dimensiones centrales para el desarrollo de la 
Madariaga, Aurora; Romero, Sheila; Romera, Liana y Lazcano, Idurre

Personas jóvenes con discapacidad y el papel que juega la familia en su ocio

conducta autodeterminada en las personas con discapacidad. En M. F. Peralta, M. C. González y C. Iriarte (Coords.). Podemos hacer oír su voz. Claves para promover la conducta autodeterminada (pp. 127-166). Aljibe.

Grigal, M., Neubert, D. A., Moon, M. S., y Graham, S. (2003). Self-determination for students with disabilities: Views of parents and teachers. Exceptional Children, 70(1), 97-112. https://doi. org/10.1177/001440290307000106

Hodapp, R. y Fidler, D. J. (1999). Parenting, etiology, and personality-motivational functioning in children with mental retardation. En E. Zigler y D. BennettGates (Eds.). Personality development in individuals with mental retardation (pp. 206-225). Cambridge University Press.

Iso-Ahola, S. E. (1980). The social psychology of leisure and recreation. W. C .Brown Company Publishers.

Lazcano, I., Madariaga, A., y Lázaro, Y. (2014). La vertiente objetiva y subjetiva de la experiencia de ocio de las personas jóvenes con discapacidad en Euskadi. En M. G. Pérez y A. De Juanas (Eds.), Construir futuros en tiempos de crisis (pp. 457-472). UNED y Grupo de Investigación de Intervención Socioeducativa en Contextos Sociales.

Lila, M., Musitu, G., y Buelga, S. (2000). Adolescentes colombianos y españoles: diferencias, similitudes y relaciones entre la socialización familiar, la autoestima y los valores. Revista Latinoamericana de Psicología, 32(2), 301-319.

Madariaga, A., Lazcano, I., y Doistua, J. (2014). Oferta comunitaria de ocio: Un análisis descriptivo desde las necesidades de los jóvenes con discapacidad. En G. Pérez y A. De Juanas (Eds.), Educación y jóvenes en tiempos de cambio (pp. 419-432). UNED y Grupo de Investigación de Intervención Socioeducativa en Contextos Sociales.

Madariaga, A., y Lazcano, I. (2014). Estudio sobre la situación del ocio de la juventud con discapacidad en Bizkaia. Diputación Foral de Bizkaia.

Madariaga, A., y Romero, S. (2016). Barreras percibidas entre los jóvenes para no participar en actividades de ocio. Revista de Psicología del Deporte, 25(S-2), 21-26.

Martínez, A. E., Inglés, C. J., Piqueras, J. A., y Ramos, V. (2010). Importancia de los amigos y los padres en la salud y el rendimiento escolar. Electronic Journal of Research in Education Psychology, 8(1), 111-138.

Molina, M. C., Mateos, A., y Violant, V. (2015). Estilos de vida saludables de adolescentes acogidos en familia extensa. Revista de Ciencias Sociales (Ve), XXI(4), 479-493.

Neulinger, J. (1980). The psychology of leisure. Charles C. Thomas Publisher.

Ortega, P., Torres, L. E., Reyes, A., y Garrido, A. (2010). Paternidad: Niños con discapacidad. Revista Intercontinental de Psicología y Educación, 12(1), 135-155.

Peñafiel, F. (2012). Planteamiento inclusivo de la participación de la familia en contextos de intervención con alumnos con discapacidad. Revista de Educación Inclusiva, 5(2), 117-128.

Peralta,F.(2008).Educaren autodeterminación: Profesores y padres como principales agentes educativos. Educación $y$ Diversidad. Revista Inter-universitaria de Investigación sobre Discapacidad e Interculturalidad, (2), 151-166.

Peralta, F., y Arellano, A. (2010). Familia y discapacidad. Una perspectiva 
teórico-aplicada del enfoque centrado en la familia para promover la autodeterminación. Electronic Journal of Research in Educational Psychology, 8(3) 1339-1362.

Portacio-Díaz, A., Sahagún-Navarro, M., y Vales-Hidalgo, A. (2020). Necesidades y apoyos percibidos por personas con diversidad funcional visual en Sucre, Colombia. Revista de Ciencias Sociales, 26(1), 175-186. https:// dx.doi.org/10.31876/rcs.v26i1.31318

Romero, S., Madariaga, A., y Lazcano, I. (2014). Principales características de la práctica deportiva de los jóvenes vascos con discapacidad. En G. Pérez y A. De Juanas (Eds.), Educación y jóvenes en tiempos de cambio (pp. 485493). UNED y Grupo de Investigación de Intervención Socioeducativa en
Contextos Sociales.

Sánchez, P. (2006). Discapacidad, familia y logro escolar. Revista Iberoamericana de Educación, 40(2), 31-41. https:// doi.org/10.35362/rie4022524

Soto, M., Valdez, L. M., Morales, D., y Bernal, N. (2015). Niveles de resiliencia, adaptación y duelo en padres de familia ante la discapacidad. Revista Mexicana de Medicina Física $y$ Rehabilitación, 27(2), 40-43.

Wehmeyer, M. L., y Field, S. L. (2007). SelfDetermination: Instructional and assessment strategies. Corwin Press.

Zulueta, A., y Peralta, F. (2008). Percepciones de los padres acerca de la conducta autodeterminada de sus hijos/as con discapacidad intelectual. Siglo Cero, 39(1), 31-43. 